\title{
A study of remotely booking slot for vehicle using Internet of Things
}

\author{
S. Metilda Florence ${ }^{1}$, M. Uma ${ }^{2}$, C. Fancy ${ }^{3}$, G. Saranya ${ }^{4}$ \\ ${ }^{1,3,4}$ Department of Information Technology, SRM Institute of Science and Technology, India \\ ${ }^{2}$ Department of Software Engineering, SRM Institute of Science and Technology, India
}

\begin{tabular}{l}
\hline Article Info \\
\hline Article history: \\
Received Sep 18, 2019 \\
Revised Apr 17, 2020 \\
Accepted May 1, 2020 \\
\hline
\end{tabular}

Keywords:

Internet of things

iParking

Open service gateways

RFID

Raspberry Pi

\begin{abstract}
Internet Of Things (IoT) is a continually growing area which aids us to unite diverse objects. The proposed system exhibits the universal notion of utilizing cloud-based intellectual automotive car parking facilities in smart cities as a notable implementation of the IoT. The RFID technology is used in this system along with suitable IoT protocols to evade human interference, which reduces the cost. Information is bartered using readers and tags. Open Service Gateways can be effectively used for this module. This system established on the consequence of IoT and the purposes are solving the chaos, bewilderment, and extensive backlogs in parking spaces. The proposed work aims to solve these problems and offer car drivers a hassle-free and instantaneous car parking experience. A Raspberry Pi would act as a mini-computer in our system. A suitable smallest path methodology would be cast-off to obtain the shortest distance between the user and every car park in the system. Hence, the pausing time of the user is decreased. This work furthermore includes the practice of remotely booking of a slot with the collaboration of android application exercising smartphones for the communication between the Smart Parking system and the user.
\end{abstract}

Copyright $₫ 2020$ Institute of Advanced Engineering and Science. All rights reserved.

\section{Corresponding Author:}

S. Metilda Florence,

Department of Information Technology,

SRM Institute of Science and Technology,

Tamil Nadu, India.

Email: metildam@srmist.edu.in

\section{INTRODUCTION}

As an essential element of a smart city application, smart parking is a good representation of how the IoT widely employed in our daily living surroundings to render various support to various users. Smart parking could be transformed for instance to allow a driver to get a smart city application over a Internet or mobile in assistance to track and store a parking spot in a city's mall based on some inclinations (e.g., near the entrance of the railway station) and organize for the parking spot spending ones credit/debit card. Models used for car parking system can be: parking gateway monitoring and parking lot examining. In the parking gateway, entries/exits and to/from details needs to be taken care of as this data is suitable to provide valuable parking information to the user. Availability of spaces and reservations would be possible is that the data mentioned above is provided. Sensors would be present around suitable places to identify the presence/absence of vehicles, and this data can be mapped, which would be further used for parking guidance. To enhance the parking facilities, this paper introduce a new system architecture based on IoT and mathematical model.

This method suggests a car park network, where evey car parking is a node in a network. That node acquires the facts from the neighboring node, thereby ensuring smooth vehicle movement at a low cost and increasing the likelihood of finding a free parking lot. Compared with other parking systems this system 
achieves better performance. This system's performance is tested through simulation and implementation. The proposed system decreases the number of cars that struggle to find a parking space and minimizes the cost of moving to the parking lot. The cost described here is the time the user has to wait for the service, thus helping users save time, save money and reduce pollution.

\section{RELATED WORK}

A smart parking system indicates the user's closest parking slots. It shows only short driving distance as sharing norm [1]. A different model based on IoT [2] takes help of camera and Raspberry-pi to uninterruptedly take pictures of the parking slot to find unoccupied ones. Another method has been recommended by crafting the performance of Android-based applications. The car can map the distance to/from the gate using the application. Microcontrollers are present on the gate are help the car to interact, and monitor the free parking slot. Upon finding an empty slot, the car can track that slot and find a suitable route towards the parking spot [3]. A different RFID based paper allocates parking space to vehicles in the parking lot when any suitable space is existing in that area. Incrementation and reduction happen automatically once a car enters or leaves that space [4]. Another author Samaras et-al used something that is called KATHODIGOS, which makes use of wireless technologies and a variety of wireless applications to obtain knowledge concerning the position of the parking slots along the roads. The collected information is now sent using gateways to a principal information system [5]. In parking management, termed 'iParking,' that observes the incoming/outgoing vehicles with the help of a sensor network. This 'iParking' helps in finding the correct parking slots and gives information to the user [6].

The writers offer a technique that takes the help of image detection [7-12]. Started with the base station (BS) positioning challenge, the optimum position of nodes in a mesh link system has improved to a wireless sensor network topology focused optimization with results ranging from dynamic programming to genetic algorithms [13-15] and further towards Tabu search [16, 17]. If we focus around the data obtained from the sensors in topography using sight of data broadcast, the work offered is breaking the observed topography into smaller units called cells and then examines how different sensors must be distributed throughout the cells in the most efficient way [18-20]. The papers are focused on an experimental explanation using a multi-opinionated evolutionary optimization [21-23]. In the situation, the problem is calculated as a non-linear optimization situation which is resolved with a self-incremental algorithm. This algorithm places nodes one by one into the positioned network is the most proper way possible [24].

\section{METHODOLOGY}

\subsection{Dynamic slot allocation}

Smart parking systems can result in the effective lowering of traffic in and around the city to a large extent. For remote usage, the user can access the system with the help of an application which can able to run in any platform irrespective of android, iOS, Blackberry, or something else. The application can be easily installed in the Smartphone or else a personal computer version can also be established. The system's primary database lies in the cloud; hence, the software requires some web services to access the system's full potential. Web services could be availed by any network such as $2 \mathrm{G}, 3 \mathrm{G}, 4 \mathrm{G}$, and so on. The Open Service Gateway functions as the link between the cloud tier and mobile applications. The OSGi (Open Service Gateway Initiative) shown in Figure 1, establishes a scenario where the clustering is easily possible and as a result of which web applications can be suitably separated into groups. It helps in describing the service as generated by the user and then returns its behavior. A key-value focused database can be adapted in this tier to ensure expandability and disseminate job queues, and also to provide performance at high level and car parking services for users on demand. A load balancer can be used to distribute the user's requests across the full range of web servers to improve the web resources' utilization.

Using distributed system, log data from web server are collected and forwarded to the cloud. E.g., when a user comes nearer to the Shopping Mall, the application automatically sent a request on account of the client to an OSGi car parking server requesting for a suitable parking space. Based on the user's comfort defined in the user profile, the web server locates the well suited vacant parking lot for this user and reserves it (optionally).

The user then can navigate to that location as driving routes with a complete map are being provided to the user by the application, by employing the Google Maps app with an android API. Distributed log data are collected with high speed data pipe. The other two tiers are linked by OSGi web servers. A variety of car parking assistance can be implemented, which are defined by their service descriptions (SD). LibeliumMetiora Smart parking sigfox kit can be utilized for installation of the nodes as it's advantageous magnetic 
field sensor (3-axis), small and weatherproof enclosure, or road- surface easy placement, default runs seemingly fast (no programming needed), remote manager and prolonged battery life.

Open hardware like Raspberry $\mathrm{Pi}$ and Arduino can be used using proper IoT protocols. An ultrasound sensor would be connected to the open hardware and a suitable WiFi module for the reception of data to a server. Protocols such as CoAP (Constrained Application Protocol) and MQTT (Message Queue Telemetry Transport) can be used for data transfer, and then the gathered data is then updated in real-time to the Web application. In other scenario where RFID reader scans the tag present on the car plate, Arduino can be used to gather this info and collective process to provide with essential information as the total number of parking spaces, percentage of free spaces, a mini-map, status of checking, etc. Once the vehicle enters the parking lot, the count would be increased and decreased upon departure.

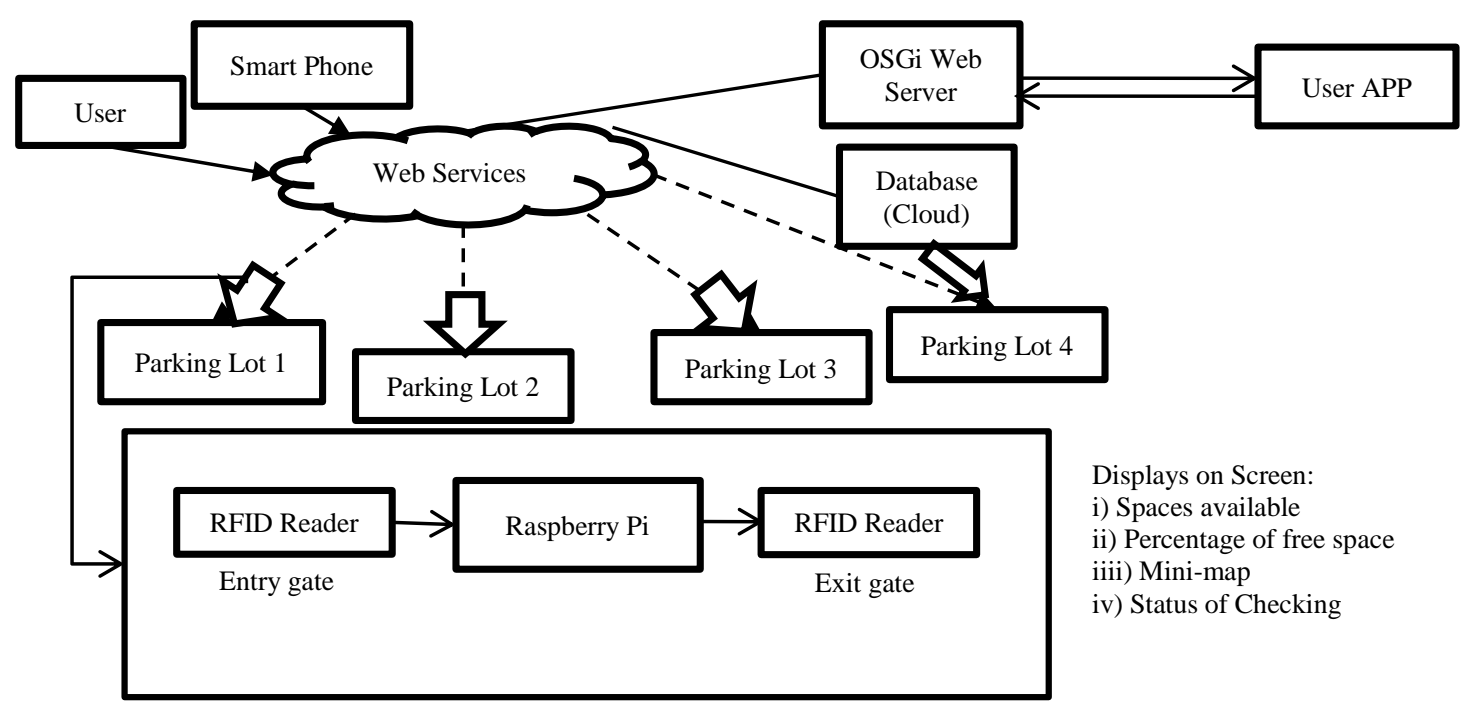

Figure 1. Proposed architecture of IoT based smart car parking system

As depicted in Figure 1, connections of Raspberry Pi can be seen. It can act as servers in the IoT smart parking architecture. The proposed device for this system would be Raspberry Pi, which is a mini computer and would result in more effectiveness than Arduino, which only acts as a subset of Raspberry Pi. The Constrained Application Protocol (CoAP) is a focused web transfer protocol to be used with constrained nodes and constrained networks in the Internet of Things. This application protocol, which is a lightweight alternative to HTTP and works on top of UDP, can be used in wireless sensor nodes (WSN). An ultrasonic sensor is connected to a Raspberry pi helps in detecting the occupancy of the slot. Raspberry pi has an IoT framework called WebIOPi which includes a CoAP server. For wireless networking, the built-in WiFi module of Raspberry pi can be utilized. After receiving the suitable data, the message is sent to the client.

\subsection{Service models}

The entire system can be noted as a service queue using the simple concept of FIFO (first in first out) [25]. First, the user has to enter the park. For the entering process, FIFO and a Markovian process work hand in hand. A Markov process is a random process which generates future possibilities taking in note its most recent activities. The mathematical model for the ingoing process can be portrayed as follows: $\mathrm{MK} / \mathrm{MK} / \mathrm{l} / \mathrm{S} / \mathrm{FIFO}$. Here, the first " $\mathrm{MK}$ " indicates that the allocation of the arrival process is Markovian (Poisson distribution), the second " $\mathrm{MK}$ " stances for the service time distribution, which is also a Markovian process (exponential distribution), " 1 " here represents the server, and $\mathrm{S}$ is the number of lots. $\mu \mathrm{I}$ is the inter-arrival time amongst two users, and $\mu \mathrm{J}$ is the tune-up time (generally the parking time). By assuming that $\mu \mathrm{J}>\mu \mathrm{I}$, the queue does not nullify. For this queue $(\mathrm{MK} / \mathrm{MK} / 1)$, the average waiting time is expressed as

$$
T_{a}=\frac{\mu_{J}^{2}}{\mu_{J}-\mu_{I}}
$$


For this queue $(\mathrm{MK} / \mathrm{MK} / \mathrm{S})$, the average waiting time is

$$
T_{a}=\frac{\mu_{J}^{2}}{S\left(\mu_{J}-\mu_{I}\right)}
$$

Hence, the average pausing time of the arrangement is

$$
\overline{T_{a}}=\frac{\sum_{i=1}^{M} T_{a_{i}}}{M}
$$

where $\mathrm{M}$ is the total amount of parking spaces.

The workflow of the proposed system is given as follows: Initially the user is required to $\log$ into the system. A request is automatically sent for wanting of a parking space. If the spaces in that particular lot are not available, then the user is forwarded to the nearest parking lot. In this network, each car parking area is working as a node, and vehicles are parked in these nodes with the help of sensing mechanisms. Each node in this system acquires the essential information from the neighboring node, consequently leading to smooth traversing of cars at minimal cost and improving the chances of locating a free parking area. As the vehicle enters, it sends a request to the system, and the system in return searches the best car park. A response is generated and reflected to the user. After successful authentication, the user is able to find its suitable location in the system. Messages are being sent to the user at every specified interval.

\subsection{Entry-parking lot}

As shown in Figure 2, the RFID reader scans the tag upon arrival of the car at the parking lot entry. Corresponding data with relevance to the approached car is then checked for in the database. The user's account details would be synced with the car's tag. The system would check for the availability of parking spaces in the parking lot. If it is greater than 0 , the system will continue to the later stages; otherwise, a message saying that space is not available would be shown to the user. Later stage involves checking the booking status. If the parking area is already booked over the web or through the application, then the parking slot will be displayed on the screen along with a notification. Navigation would also be provided. If the booking is not confirmed, then the system would check for the availability of parking spaces. On finding a suitable area, the amount would be calculated, and space would be provided along with a notification and directions for navigation. If an area is not available, a message saying the same would be displayed to the user.

\subsection{Exit parking- lot}

Once the car is ready to leave the parking spot, an RFID scanner scans the tag at the exit-gate and calculates the amount once again to ensure accuracy, as depicted in Figure 3. The generated amount is then displayed on the screen. The user can pay for the service at the same time or later within a specific period. If he chooses the latter, the data is uploaded in the cloud, and also his bank details are saved. If payment is not made within a mentioned time frame, then the amount would be charged automatically from the user. If the balance is insufficient, then the user is asked to recharge their account (Smart parking app account) and proceed accordingly. Upon recharging, the entire global database is updated.

\section{IMPLEMENTATION}

IR sensors are placed in the parking lots to determine whether a parking slot is vacant or not. The Raspberry Pi is wirelessly connected with IR sensors using the ESP (Espressif modules) chip. An ESP chip is used to access a WiFi network. Raspberry Pi acts like intermediate processing unit between the sensors and cloud. The status of various analog input pins on a Raspberry Pi chip are checked by a $\mathrm{C}$ language script which is running on the chip and updates this information onto the cloud. From various sensors, the collected data is sent to the Raspberry Pi through the ESP chip. The Raspberry Pi then transfers this data to MYSQL Server. RFID tag is being teamed up with the android application. RFID reader is used to scan the car tag upon entry and exit of the car at the parking area. 


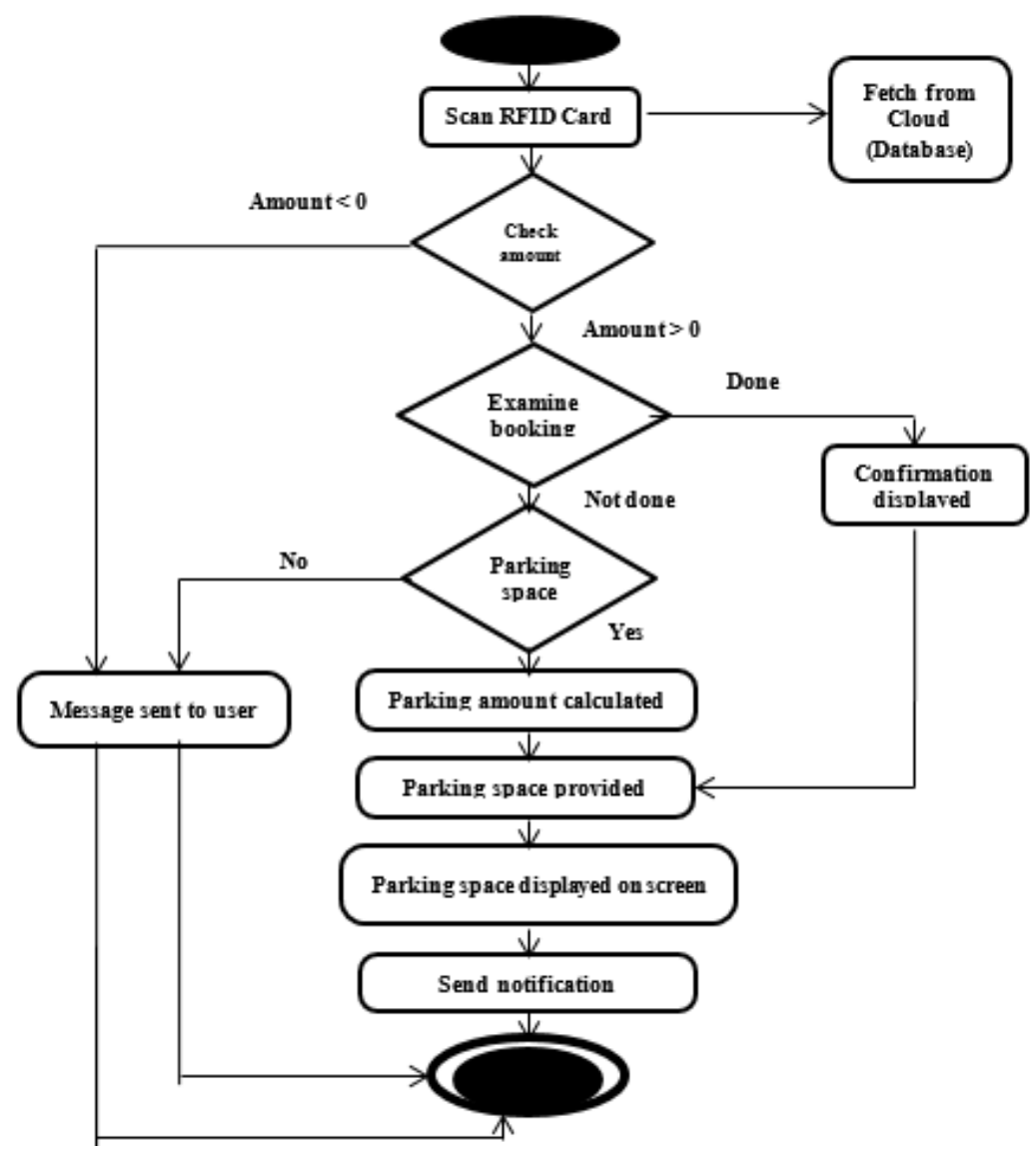

Figure 2. Entry-parking lot

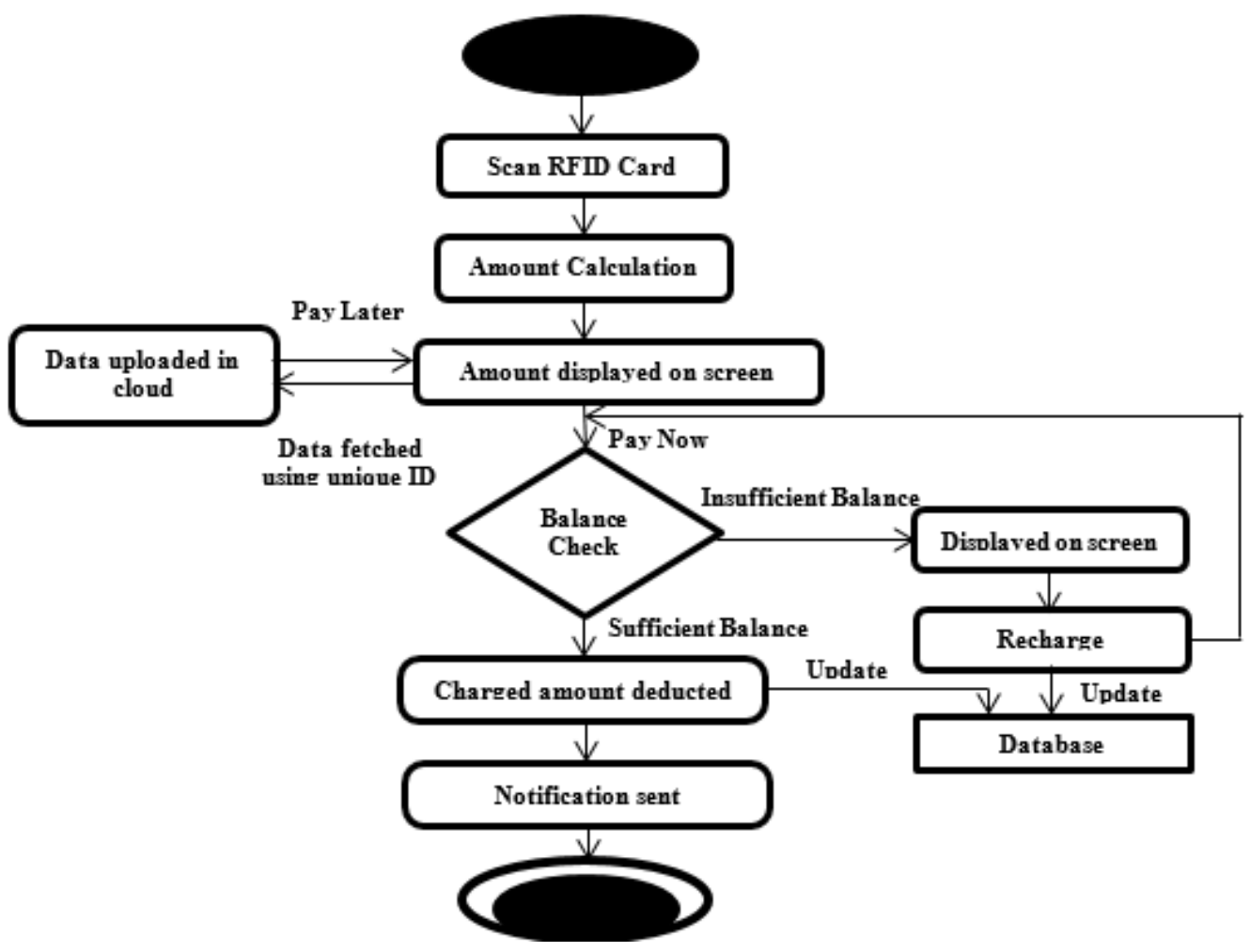

Figure 3. Exit-parking lot 


\section{RESULT AND DISCUSSION}

To start with Smart parking App must be installed on our mobile. After installation, when the user clicks the Smart Parking App, Login screen will be displayed as in Figure 4(a). After providing registered credentials correctly, the App will display the available slots in white color and booked slot in red color shown in Figure 4(b). Selected parking slot will change its colour and popup will be displayed as shown in Figure 4(c). After confirmation, the slot will be booked. We can also cancel the booking slot as shown in Figure 4(d). This App providing navigation route map from current location to near by parking area, which is depicted in Figure 4(e). Using registered RFID card the user can make payment while exiting the parking.

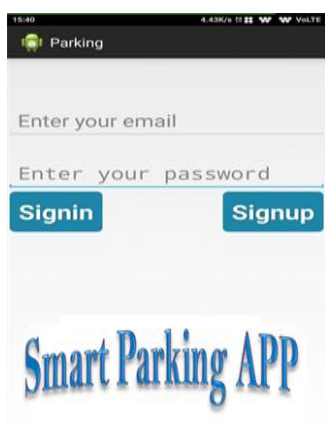

(a)

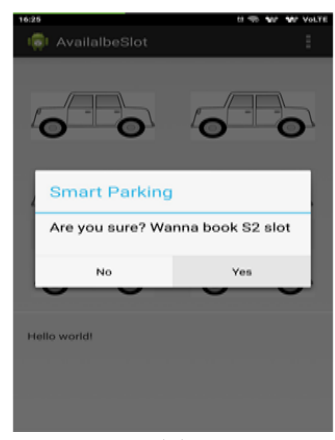

(c)
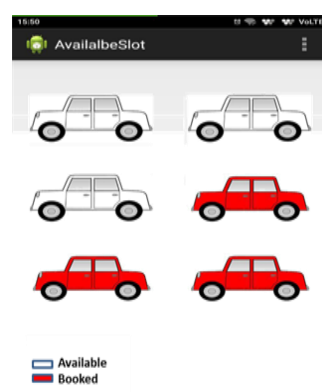

(b)

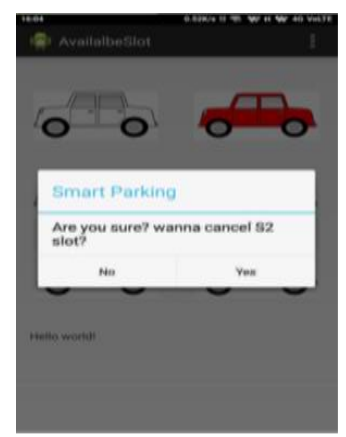

(d)

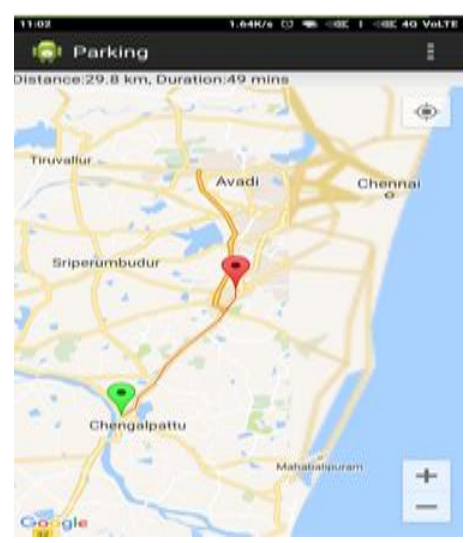

(e)

Figure 4. Screen shots of smart parking system application, (a) login screen, (b) showing availability of slots, (c) booking slot, (d) cancellation of slot, (e) navigating map

\section{CONCLUSION AND FUTURE ENHANCEMENT}

Productive performance of this work would be the outcome in traffic and disorder in jam-packed parking spaces like stations, airports, malls, stadiums where a conventional parking space is shared. Since everything would be automatic, therefore traveling without cash can be seemingly possible. It would help in reducing the long queues, waiting time, stress, tension, and result in increasing the capacity of 
the system to a fruitful ground. Also, the system requires negligible human resources, as a result of which human errors can be totally avoided, and security can be advanced, which would result in an amazing and swift experience for the user. Sensors, communication, and the application layer are the fundamentals for this system. Effective node placement is the key, along with the effectual utilization of the algorithm can help us in achieving milestones. RFID is being teamed up with the android application to provide the best user experience for the control systems and vehicles. If the conditions are properly met, this will result in an effective reduction of the waiting times. The ideal solution is provided by the planned system, where the maximum of the vehicles locates able parking space successfully.

Better performance, effective large scale parking, and low cost are being met by this system. Various kind of data structure algorithms can be examined properly in order to find a suitable combination that would satisfy the needs of this system. The concept of queuing can be implemented if the number of vehicles at a specific location exceeds the threshold. Then these vehicles can be easily redirected to the next nearest parking location where the user can park his/her car with ease and at the same time ensuring safety and ease. This system can easily achieve proper authorization and location of the spot. Applications in the future will run on any platform. For roadside parking, hour slot booking can be established. At times, proper parking slot is not available along the roadsides, so user faces a lot of discrepancy and chaos to find a parking slot. Also if a parking lot is not available nearby, then the user is forced to park the car in the roadside. For this, sensors can be placed along the roads, and time slots can be given to the user. The software interface will show after what time a particular slot might be available in that region. Hence it will provide information to the user as to when he should arrive at that location in order to attain that space. Space could be quickly booked online, with the help of sensors and cameras. The user is able to specify the duration of hours for which he/she wishes to utilize that parking space. This would help in notifying other such customers who would wish to park their cars in that neighborhood.

\section{REFERENCES}

[1] V. Hans, et al., "An approach to IoT based car parking and reservation system on Cloud," 2015 International Conference on Green Computing and Internet of Things (ICGCIoT), pp. 352-254, 2015.

[2] Basavaraju S. R., "Automatic Smart Parking System using Internet of Things (IOT)," International Journal of Scientific and Research Publications, vol. 5, no. 12, pp. 629- 632, 2015.

[3] D. J. Bonde, et al., "Automated car parking system commanded by android application," International Journal of Computer Science and Information Technologies, vol. 5, no. 3, pp. 3001-3004, 2014.

[4] E. Soni, et al., "Design and Development of RFID Based Automated Car Parking System," The International Journal of Mathematics, Science, Technology and Management, vol. 2, no. 2, pp. 6-8, 2016.

[5] A. Samaras, et al., "A Novel Smart Parking System based on Wireless Sensor Networks," in Proceedings of the 1st International Virtual Conference on Intelligent Transportation Systems, Slovakia, pp. 140-145, 2013.

[6] J. Chinrungrueng, et al., "IParking: A parking management framework," in Proceedings of the 11th International Conference on ITS Telecommunications (ITST), St. Petersburg, Russia, pp. 63-68, 2011.

[7] A. A. Yass, et al., "New design for intelligent parking system using the principles of management information system and image detection system," in Proceedings of the 2009 International Conference on Computer Engineering and Applications, vol. 2, pp. 360-364, 2011.

[8] D. B. L. Bong, et al., "Integrated approach in the design of car park occupancy information system (COINS)," IAENG International Journal of Computer Science, vol. 35, no. 1, pp. 7-14, 2008.

[9] L. Atzori, A. Iera, and G. Morabito, "Understanding the Internet of Things: definition, potentials, and societal role of a fast evolving paradigm," Ad Hoc Networks, vol. 56, pp. 122-140, 2017

[10] Jayakshei Dadaji Bachhav, Mechkul M.A, "Smart car parking system," International research Journal of Engineering and technology, vol. 4, no. 6, pp. 3036-3038, Jun. 2017

[11] Fengli Zhou, and Qing Li, "Parking Guidance System Based on ZigBee and Geomagnetic Sensor Technology," 13thInternational Conference on Distributed Computing and Applications to Business, Engineering and Science (DCABES), IEEE, pp. 268-271, Nov. 2014.

[12] Hong Wang, and Wenbo He, "A Reservation-based Smart Parking System," IEEE Conference on Computer Communications Workshop, pp. 690-695, 2011

[13] R. Bose, "A smart technique for determining base-station locations in an urban environment," IEEE Transaction on Vehicular Technology, vol. 50, no. 1, pp. 43-47, 2001.

[14] J. K. Han, et al., "Genetic approach with a new representation for base station placement in mobile communications," in Proceedings of the 2001 IEEE Vehicular Technology Conference, Atlantic City, NJ, USA, vol. 4, pp. 2703-2707, 2001.

[15] Prabhu Ramaswamy, "IoT Smart Parking System for Reducing Green House Gas Emission," 2016 Fifth International Conference On Recent Trends In Information Technology, pp. 1-6, 2016.

[16] Jae Kyu Suhr and Ho Gi Jung, "Sensor Fusion-Based Vacant Parking Slot Detection and Tracking," in Intelligent Transportation Systems, IEEE, pp. 21-36, 2014. 
[17] H. Meunier, et al., "A multiobjective genetic algorithm for radio network optimization," in Proceedings of the 2000 Congress on Evolutionary Computation, La Jolla, CA, USA, vol. 1, pp. 317-324, 2000.

[18] E. Amaldi, et al., "UMTS radio planning: Optimizing base station configuration," in Proceedings of the 2002 IEEE Vehicular Technology Conference, Vancouver, BC, Canada, vol. 2, pp. 768-772, 2002.

[19] Masahiro Kenmotsu, Weihua Sun, Naoki Shibata, Keiichi Yasumoto and Mi-noru Ito, "Parking Navigation for alleviating Congestion in Multilevel Parking Facility," in Vehicular Technology Conference (VTC Fall), IEEE, pp. 1-5, 2012.

[20] H. Zhang and J. C. Hou, "On the upper bound of $\alpha$-lifetime for large sensor networks," ACM Transactions on Sensor Networks, vol. 1, no. 2, pp. 272-300, 2005.

[21] Yang Z, and Pun-Cheng, L.S.C, "Vehicle detection in intelligent transportation systems and its applications under varying environments," A review. Image and Vision Computing, vol. 69, pp. 143-154, 2018

[22] C. M. Fonseca and P. J. Fleming, "Genetic algorithms for Multiobjective Optimization: Formulation, Discussion and Generalization," in Proceedings of the Fifth International Conference on Genetic Algorithms, UrbanaChampaign, IL, USA, vol. 93, pp. 416-423, 1993.

[23] D. B. Jourdan and O. L. de Weck, "Multi-Objective Genetic Algorithm for the Automated Planning of a Wireless Sensor Network to Monitor a Critical Facility," in Proceedings of the Sensors, and Command, Control, Communications, and Intelligence (C3I) Technologies for Homeland Security and Homeland Defense III, Orlando, FL, USA, vol. 5403, pp. 565-575, 2004.

[24] W. Li and C. G. Cassandras, "A minimum-power wireless sensor network self-deployment scheme," in Proceedings of the 2005 IEEE Wireless Communications and Networking Conference, New Orleans, LA, USA, vol. 3, pp. 1897-1902, 2005.

[25] Y. Geng and C. G. Cassandras, "A new smart parking system based on optimal resource allocation and reservations," in Proceedings of 14th International IEEE Conference on Intelligent Transportation Systems (ITSC), pp. 979-984, 2011.

\section{BIOGRAPHIES OF AUTHORS}
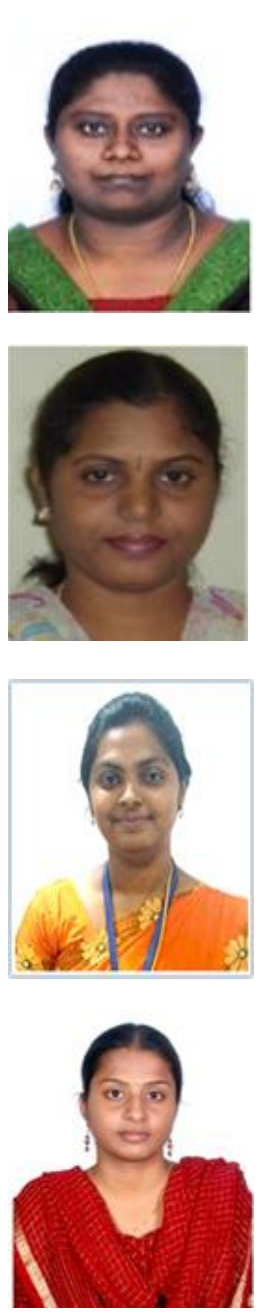

Dr. S. Metilda Florence, completed $\mathrm{PhD}$ in Video Processing area from Bharathiar University, Coimbatore, India. Received M.Tech degree in Computer Science from SRM University, Chennai, India and MCA from Bharathidasan University, Trichy, India. She has 18+ years of teaching and research experience. Currently she is an Assistant Professor (Senior Grade) in Information Technology Department at SRM Institute of Science and Technology, India. She is the author of 10 International journals and 8 International conference papers. Her research interests include Image processing, Data mining, Recommendation system, Machine learning and deep learning.

Dr. Uma Mohan, received an M.Tech in Computer Science from SRM University, Chennai, India, an MCA from Bharathidasan University, Trichy and is completed her Ph.D in the area of Brain Computer Interface from Bharathiyar University, Coimbatore, India. She has 18+ years of teaching and research experience. Currently, she is an assistant professor (Selection Grade) in Department of Software Engineering, SRM Institute of Science and Technology, India. She is the author of 18 journal publications and 17 national and international conferences. Her research interests include brain computer interface,P300, Data Mining, Personalization, Recommendation System, Machine learning, Web services and Data Science.

Ms. C. Fancy is currently working as an Assistant Professor at Department of Information Technology, SRM Institute of Science and Technology, Kattankulathur. She is currently pursuing doctoral research in Data center networking. Her research interests include Software Defined Networks, Image Processing, Cryptography, Network security and Cloud computing.

Ms. G. Saranya, received B.Tech degree in Information Technology from Sri Venkateswara College of Engineering, Sriperumbudur, Chennai, India in 2009. M.Tech degree in Information Technology from Vel Tech Multi Tech Dr.Rangarajan Dr.Sakunthala Engineering College, Avadi, Chennai, India in 2011. She is currently pursuing her Ph.D in Computer Science and Engineering at Sathyabama Institute of science and Technology, Chennai, India and works as Assistant Professor in Information Technology Department at SRM Institute of Science and Technology, India. She has $7+$ years of teaching experience and 2 years of Industry Experience. Her research interests include Big Data Analytics, Machine learning and Deep learning. 